\title{
Frame-based Gaussian beam shooting simulation of back-scattered fields in the presence of a building
}

\author{
E.A. Fnaiech* \\ C. Letrou $^{\dagger}$
}

\begin{abstract}
In the context of ground based Radar or wireless communications, non line of sight situations occuring in suburban type built environments are particularly demanding regarding the accuracy of field simulations. This communication presents new developments of the frame-based Gaussian beam shooting method to address some of the diffraction problems encountered in the presence of buildings.

The presentation is focused on the problem of back-scattering by the bottom and the top of a building, which is addressed through incident beam selection, image frame and frame redecomposition. Numerical results obtained with this algorithm are compared to results obtained with the Parabolic Equation and with reference results.
\end{abstract}

\section{INTRODUCTION}

The Gaussian Beam Shooting (GBS) method is well suited to $3 \mathrm{D}$ field simulations in multi-reflecting contexts due the paraxial approximation of reflected fields, leading to tracking algorithms similar to ray-based ones. In the millimetric range, such "paraxial tracking" GBS algorithms were validated by comparison with experimental results, in indoor environments [1]. In urban or suburban environments however, at frequencies of conventional communication or Radar systems, paraxial Gaussian beam (GB) tracking is unable to account for diffraction effects with reasonable accuracy. Simulation of diffracted fields in the form of GB summations ("beam to beam" formulations) is thus a bottleneck for efficient use of GBS in such contexts.

Only few recent studies have focused on this problem, leading to different formulations, each of them specific to the method used to discretize the radiated and scattered fields into sets of GBs: empirical sampling and normalisation at arrival [2], empirical sampling in the angular domain and frame decomposition along the edge [3], frame decomposition and frame based truncation of fields on obstacle surface [4]. The latter approach is used in this work.

*Télécom SudParis (Lab. SAMOVAR - UMR CNRS 5157), 9 rue Charles Fourier, 91011 Evry Cedex, France, e-mail: @telecom-sudparis.eu, tel.: +33 1607644 34, fax: +33160764433.

${ }^{\dagger}$ Télécom SudParis (Lab. SAMOVAR - UMR CNRS 5157), 9 rue Charles Fourier, 91011 Evry Cedex, France

$¥$ NOVELTIS, 153 rue du Lac, 31670 Labege, France

$\S$ THALES Air Systems S.A., Hameau de Roussigny, 91470 Limours, France

\author{
A. Ginestet ${ }^{\ddagger}$ \\ G. Beauquet ${ }^{\S}$
}

Section 2 is devoted to an outline of the frame based GBS algorithm in free space and of paraxial Gaussian beam tracking in the presence of reflecting surfaces, introducing the image frame concept. Section 3 presents some elements of a specific "beam selection" algorithm, which allows to know which beams are incident on a given obstacle, and recalls the principle of the frame redecomposition algorithm used to cope with diffraction. Section 4 presents a comparison of results obtained by GBS over a reflecting ground with Parabolic Equation simulations and Method of Moments results.

\section{FRAME BASED GAUSSIAN BEAM SHOOTING AND PARAXIAL TRACKING}

In GBS and tracking algorithms, propagated fields are represented as a superposition of Gaussian beams (GB), which are launched from the emitting antenna and transformed through successive interactions with obstacles. The formulation developed in this work is based on frame decomposition of source fields: a planar distribution of source fields is expressed as a weighted sum of Gaussian windows which form a "frame", and radiate each in the form of a Gaussian beam.

\subsection{Frame of Gaussian windows}

The frame windows $\Psi_{\boldsymbol{\mu}}(y, z)$ used to describe a planar source distribution in the $y O z$ plane are constructed as the product of frame windows $\psi_{m, n}^{y}(y)$ and $\psi_{p, q}^{z}(z)$ in $L_{2}(\mathbb{R})$ :

$$
\Psi_{\boldsymbol{\mu}}(y, z)=\psi_{m, n}^{y}(y) \psi_{p, q}^{z}(z)
$$

where $\boldsymbol{\mu}=(m, n, p, q)$ is a composite translation index in $\mathbb{Z}^{4}$.

The frame windows $\psi_{m, n}^{y}(y)$ are obtained by translation of a Gaussian function $\psi(y)$ along the spatial coordinate $y$ and along its spectral counterpart $k_{y}$ :

$$
\psi_{m, n}^{y}(y)=\psi(y-m \bar{y}) e^{i n \bar{k}_{y} y}
$$

- $\bar{y}$ and $\bar{k}_{y}$ are respectively the spatial and spectral translation steps, $m$ and $n$ the spatial and spectral translation indices. 
- $\left\{\psi_{m, n}^{y},(m, n) \in \mathbb{Z}^{2}\right\}$ is a frame if and only if $\bar{y} \bar{k}_{y}=2 \pi \nu$, with $\nu<1(\nu$ : oversampling factor).

- the favorite choice for the translation steps is: $\bar{y}=\sqrt{\nu} L, \bar{k}_{y}=\sqrt{\nu}(2 \pi / L)$ ("balanced" frame).

The same construction is used for the frame along $z$, with $p, q$ the spatial and translation indices along $z$. In the following, the Gaussian function $\psi$ is taken as:

$$
\psi(y)=(\sqrt{2} / L)^{\frac{1}{2}} e^{-\pi\left(\frac{y}{L}\right)^{2}}
$$

\subsection{Frame window radiation}

Through paraxial asymptotic evaluation of plane wave spectrum integrals, the fields radiated by the source field distributions $\Psi_{\boldsymbol{\mu}}(y, z)$ (either for $y$ or $z$ component) are put in the form of paraxial Gaussian beams. Let us denote $\boldsymbol{B}_{\boldsymbol{\mu}}^{\alpha}(\boldsymbol{r})$ the field radiated at point $\boldsymbol{r}$ by an $\alpha$-polarized, $\alpha=y, z$, frame window distribution. The paraxial expression of $\boldsymbol{B}_{\boldsymbol{\mu}}^{\alpha}$ is:

$\boldsymbol{B}_{\boldsymbol{\mu}}^{\alpha}(\boldsymbol{r})=\boldsymbol{B}_{0}^{\alpha} \sqrt{\frac{\operatorname{det} \boldsymbol{\Gamma}^{-1}(0)}{\operatorname{det} \boldsymbol{\Gamma}^{-1}\left(x_{\boldsymbol{\mu}}\right)}} \exp i k\left[x_{\boldsymbol{\mu}}+\frac{1}{2} \boldsymbol{y}_{\boldsymbol{\mu}}^{t} \boldsymbol{\Gamma}\left(x_{\boldsymbol{\mu}}\right) \boldsymbol{y}_{\boldsymbol{\mu}}\right]$

with $\boldsymbol{B}_{0}^{\alpha}$ a vector depending on the source polarization. This expression is analog to that of a geometrical optics ray along the $x_{\boldsymbol{\mu}}$ direction, with $\boldsymbol{\Gamma}$ the curvature matrix and $\sqrt{\frac{\operatorname{det} \boldsymbol{\Gamma}^{-1}(0)}{\operatorname{det} \boldsymbol{\Gamma}^{-1}\left(x_{\mu}\right)}}$ the divergence factor. $\boldsymbol{y}_{\boldsymbol{\mu}}=\left(y_{\boldsymbol{\mu}}, z_{\boldsymbol{\mu}}\right)$ is the position vector of the point $\boldsymbol{r}$ in a plane transverse to the $x_{\boldsymbol{\mu}}$ axis.

The difference between geometrical optics rays and Gaussian beams stems from the fact that $\boldsymbol{\Gamma}$ is a real matrix in the first case, a complex one in the latter. This complex matrix accounts for the Gaussian decay of fields with increasing distance from the $x_{\boldsymbol{\mu}}$ axis in transverse planes. The $x_{\boldsymbol{\mu}}$ axis is then called the beam axis.

\subsection{Frame based GBS}

Frames are complete sets hence any of the components $E_{\alpha}, \alpha=y, z$ of a source electric field distribution given in the $x O y$ plane, can be expressed as a weighted summation of the frame windows:

$$
E_{\alpha}(y, z)=\sum_{\boldsymbol{\mu} \in \mathbb{Z}^{4}} A_{\boldsymbol{\mu}}^{\alpha} \Psi_{\boldsymbol{\mu}}(y, z)
$$

with the $A_{\boldsymbol{\mu}}$ complex coefficients called "frame coefficients". These coefficients are not unique, but can be calculated by projecting the function on a "dual frame" of functions $[5,6]$.

The field radiated by an $\alpha$-polarized field distribution radiating into the $x>0$ half-space, is then obtained as the following GB summation, at a point located at $\boldsymbol{r}$ (with positive $x$ ):

$$
\boldsymbol{E}(\boldsymbol{r})=\sum_{\boldsymbol{\mu} \in \mathbb{Z}^{4}} A_{\boldsymbol{\mu}}^{\alpha} e^{i\left(m n l_{y} \kappa_{y}+p q l_{z} \kappa_{z}\right)} \boldsymbol{B}_{\boldsymbol{\mu}}^{\alpha}(\boldsymbol{r})
$$

\subsection{Image frame}

As is well-known, if a paraxial Gaussian beam is incident on a smooth reflecting surface with its "impact region" entirely on the surface (no significant field at the surface edge), its transformed field can be expressed again in the form of a paraxial Gaussian beam $[7,8]$.

When the reflecting surface is plane, this result can easily be established by considering the transformation of each plane wave of the incident beam plane wave spectrum (PWS). Introducing an "image plane" $I$, symmetric to the source plane $S$, with respect to the reflexion plane $P$, the PWS of the reflected field in that plane is of the form:

$$
\widetilde{\boldsymbol{e}}_{I}^{r}\left(k_{y_{I}}^{r}, k_{z_{I}}^{r}\right)=\overline{\mathbf{R}} \widetilde{\boldsymbol{e}}_{S}^{i}\left(k_{y_{S}}^{i}, k_{z_{S}}^{i}\right)
$$

with $\widetilde{\boldsymbol{e}}_{S}^{i}\left(k_{y_{S}}^{i}, k_{z_{S}}^{i}\right)$ the complex vector PWS of the incident beam in its source plane and $\overline{\mathbf{R}}$ the reflexion operator. Due to the Snell-Descartes law and to symmetry, and with a convenient choice of the coordinate systems $\left(O_{S}, \hat{y}_{S}, \hat{z}_{S}\right)$ and $\left(O_{I}, \hat{y}_{I}, \hat{z}_{I}\right)$ respectively in the source and image planes, the components of incident and reflected wave wavevectors verify: $k_{y_{I}}^{r}=k_{y_{S}}^{i}, k_{z_{I}}^{r}=-k_{z_{S}}^{i}$ and $k_{x_{I}}^{r}=k_{x_{S}}^{i}$.

For an incident field radiated by a frame window, $\widetilde{\boldsymbol{e}}_{S}^{i}\left(k_{y_{S}}^{i}, k_{z_{S}}^{i}\right)=\boldsymbol{f}\left(k_{y_{S}}^{i}, k_{z_{S}}^{i}\right) \widetilde{\Psi}_{\boldsymbol{\mu}^{i}}\left(k_{y_{S}}^{i}, k_{z_{S}}^{i}\right)$. Hence the reflected PWS in the image plane is of the form of a slowly varying vector function (in as much as the reflexion operator has no pole in the frame window bandwidth) multiplied by the Gaussian frame window $\widetilde{\Psi}_{\boldsymbol{\mu}^{r}}\left(k_{y_{I}}^{r}, k_{z_{I}}^{r}\right)$ where $\boldsymbol{\mu}^{r}=(m, n,-p,-q)$ if $\boldsymbol{\mu}^{i}=(m, n, p, q)$.

Defining a frame in the image plane with the same frame parameters as in the source plane, the reflected field is obtained by GBS from this plane, each GB being weighted by the frame coefficient of the corresponding source frame window.

\section{GAUSSIAN BEAM SELECTION AND DIFFRACTION}

\subsection{GB selection}

Frame based GBS starts from a complete discretization of source fields, involving a high number of frame windows. However the spatial and spectral localization of Gaussian frame windows, for a given threshold value, limits the number of launched beams which reach a region of interest. 
To this end, a specific "beam selection" algorithm has been developed, to identify for any source beam whether or not it impinges on a given plane rectangular "obstacle" or "observation area", and whether it is totally reflected by the obstacle, or incident on an edge. In the latter case, the incident beam field is "redecomposed" on a set of frame windows defined in the plane of the obstacle. In this way, the beam truncation is accounted for, similarly to what is done in Physical Optics algorithms.

Although the GB radiated by frame windows are general astigmatic, their fields (above a given threshold with respect to the beam axis field magnitude) are limited by ellipses with the same axes orientation in all transverse planes. The orientation of these axes are derived from the diagonalization of the beam complex curvature matrix in (4) [9]. The beam limits in the principal planes, containing these axes, are hyperbola.

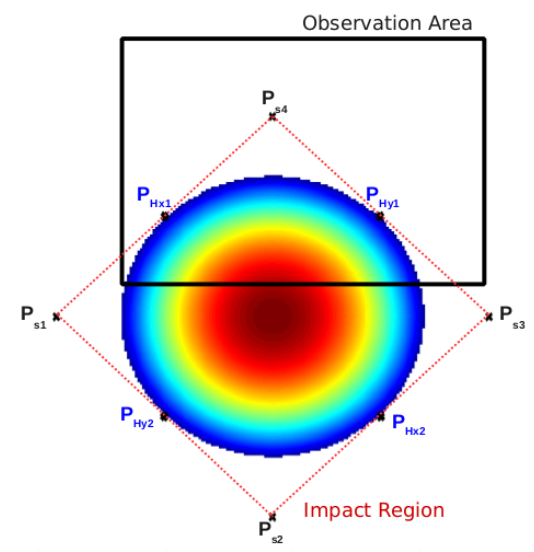

Figure 1: GB impact region in the obstacle plane. The circumscribing parallelogram is derived from the knowledge of the intersection points of the four GB limiting hyperbolas, $P_{H x 1}, P_{H x 2}, P_{H y 1}, P_{H y 2}$.

The "beam selection" algorithm is based on the computation of the intersection points of these hyperbola with the plane containing the obstacle surface, as illustrated in Fig. 1. Depending on the number of the intersection points and their positions, either beams are eliminated (no significant field in the plane of interest) or their region of impact on the plane is identified. If this region intersects the obstacle surface, the beam field is decomposed on narrow frame windows in the region of impact on the surface of the obstacle.

\subsection{Frame redecomposition}

Instead of calculating the field of all incident beams partially impinging on the obstacle, summing them and decomposing them on a local frame of spatially wide windows, in order to start GBS anew from the plane of the obstacle, a re-decomposition algorithm has been proposed. The aim of this algorithm is to provide more flexibility in the trade-off between accuracy and computational burden. This redecomposition algorithm consists in two steps:

1. decompose each incident beam field on a frame of spectrally narrow windows defined in the plane of the obstacle and sum up the frame coefficients for all incident beams; the frame coefficients of frame windows centered outside of the obstacle are not computed and put to zero, which provides the truncation effect at the edge;

2. perform a change of frame, from the narrow window frame to a wide window frame; the frame coefficients on the latter are used for GBS from the plane of the obstacle.

\section{NUMERICAL TESTS}

A number of test cases are designed to compare results obtained by GBS, with the Parabolic Wave Equation (3DPWE) and with the Method of Moments (MoM) code FEKO. The source is given as a field distribution in the $x O z$ plane, with linear polarization along $y$ or $z$. The distribution is of the form of a Gaussian frame window $\Psi_{\boldsymbol{\mu}}(y, z)$, defined by: $L=7.5 \lambda, \nu=0.16, m=n=0$ (symmetry with respect to the $x O z$ plane), $p=6, q=0$ or $q=-1$. If the $x O y$ plane is considered as the ground, the $p$ index determines the height $h$ of the source center above the ground: $h=p \sqrt{\nu} L$. In the following, the frequency is taken equal to $430 \mathrm{MHz}$, a classical frequency used for UHF ground Radar applications. For $L=10 \lambda, h=12.5 \mathrm{~m}$.

The first test case addresses the problem of propagation above a perfectly reflecting planar ground, in order to validate the use of an image frame. The results presented in Figure 2 were obtained by GBS for the horizontally polarized and tilted source $(q=-1)$. The frame used to decompose the source distribution in the $y O z$ plane was defined by $L=10 \lambda$ and $\nu=0.16$. These results appeared to compare very well with 3DPWE and MoM ones. A comparison with 3DPWE and MoM results is presented in Figure 3 along a vertical line in the $x O z$ plane, at a larger distance of $x=1000 \mathrm{~m}$, for the horizontally polarized non tilted source $(q=0)$. The width $L$ of the frame windows used to discretize the source had to be increased and was taken equal to $15 \lambda$, in order to obtain again results which compare well with the reference MoM. 


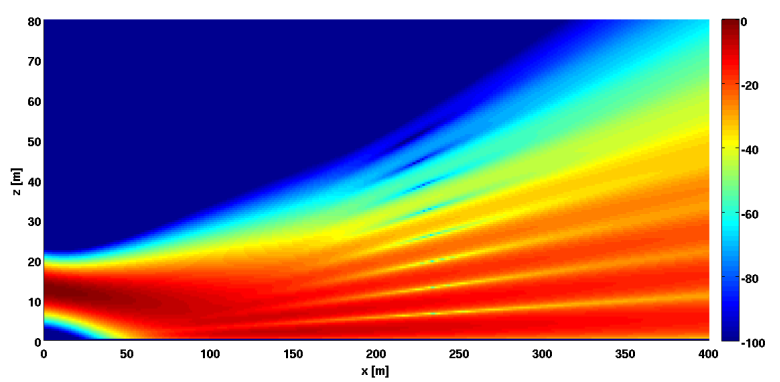

Figure 2: Propagation above a perfect metal plane, computed by GBS: horizontal field component in the vertical $x O z$ plane.

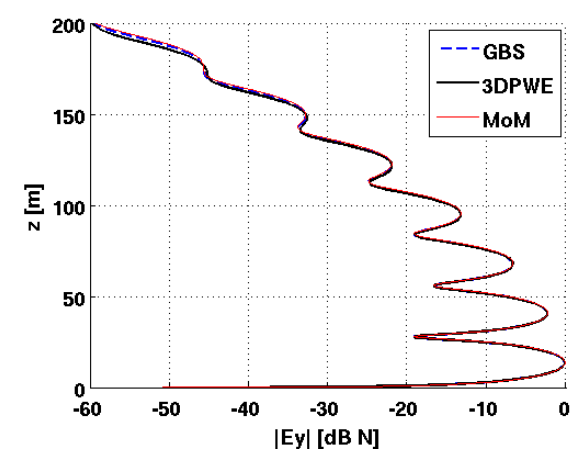

Figure 3: Propagation above a perfect metal plane, computed by GBS: comparison of the horizontal field component along the line $y=0, x=1000 \mathrm{~m}$, computed by GBS, Parabolic Wave Equation (3DPWE) and Method of Moments (MoM).

A new test case is currently under study, considering the fields backscattered by the corner between the ground and the bottom of a wall. Results will be presented at the conference.

\section{CONCLUSION}

In this work, the problem of propagation modelling in built environments is addressed with the tools provided by frame decomposition and Gaussian beam shooting. The combination of beam selection, of image frames and of beam redecomposition is proposed as a flexible tool even when obstacles are not in their respective far field. Test cases where source fields are incident on the bottom or the top of a building will be presented, with comparison between Gaussian beam shooting, Parabolic Wave Equation and Method of Moments results.

\section{References}

[1] A. Fluerasu and C. Letrou, "Gaussian beam launching for $3 \mathrm{D}$ physical model- ing of propagation channels," Annales des télécommunications- Annals of telecommunications, vol. 64, no. 11-12, pp. $763-776$, december 2009.

[2] J. M. Darras, T. George, and P. Pouliguen, "Diffraction by a sharp edge for an electromagnetic field described by summation of gaussian beams: the Ufimtsev way," in Progress in Electromagnetics Research Symposium (PIERS 2012), Moscow, Russia, August 2012.

[3] M. Katsav and E. Heyman, "Gaussian beam summation representation of beam diffraction by an impedance wedge: a 3D electromagnetic formulation within the Physical Optics approximation," IEEE Trans. Antennas Propagat., vol. 60, no. 12, pp. 5843-5858, Dec. 2012.

[4] I. Ghannoum, C. Letrou, and G. Beauquet, "Frame based Gaussian beam bouncing," in EMTS'10 : 20th International Symposium on Electromagnetic Theory, IEEE, Ed., 2010, pp. $68-71$.

[5] D. Lugara and C. Letrou, "Printed antennas analysis by a Gabor frame-based method of moments," IEEE Trans. Antennas Propagat., vol. 50, no. 11, pp. 1588-1597, 2002.

[6] A. Shlivinski, E. Heyman, A. Boag, and C. Letrou, "A phase-space beam summation formulation for ultrawide-band radiation," IEEE Trans. Antennas Propagat., vol. 52, no. 8, pp. 2042-2056, 2004.

[7] D. Lugara, C. Letrou, A. Shlivinski, E. Heyman, and A. Boag, "Frame-based Gaussian beam summation method: Theory and application," Radio Science, vol. 38, no. 2, Apr. 2003.

[8] D. Lugara, A. Boag, and C. Letrou, "Gaussian beam tracking through a curved interface: comparison with a method of moments," $M i$ crowaves, Antennas and Propagation, IEE Proceedings -, vol. 150, no. 1, pp. 49-55, 2003.

[9] D. Lugara, "Antennas and quasi-optical systems modeling using Gaussian beam decomposition (in french)," Ph.D. dissertation, INT Evry and Université de Marne la Vallée, France, Nov. 2000. 